\title{
Modeling and Validating RF-Only Interferometric Triggering with Cosmic Rays for BEACON
}

\section{A. Zeolla, ${ }^{a, *}$ S. A. Wissel, ${ }^{a, b}$ J. Alvarez-Muñiz, ${ }^{c}$ W. Carvalho Jr., ${ }^{d}$ A. Cummings, ${ }^{e}$} Z. Curtis-Ginsberg, ${ }^{f}$ C. Deaconu, ${ }^{f}$ K. Hughes, ${ }^{f}$ A. Ludwig, ${ }^{f, g}$ K. Mulrey, ${ }^{h}$ E. Oberla, ${ }^{f}$ S. Prohira, ${ }^{i}$ A. Romero-Wolf, ${ }^{j}$ H. Schoorlemmer, ${ }^{k}$ D. Southall, ${ }^{f}$ A. G. Vieregg ${ }^{f}$ and E. Zas ${ }^{c}$

${ }^{a}$ Department of Physics, Pennsylvania State University, State College, PA 16802, USA.

${ }^{b}$ Physics Department, California Polytechnic State University, San Luis Obispo, CA 93407, USA.

${ }^{c}$ Instituto Galego de Física de Altas Enerxías IGFAE Univerisade de Santiago de Compostela, 15782 Santiago de Compostela, Spain.

${ }^{d}$ Univerisade de São Paolo, Sao Pãolo, Brazil.

${ }^{e}$ Gran Sasso Science Institute, Gran Sasso, Italy.

${ }^{f}$ Department of Physics, Enrico Fermi Institute, Kavli Institute for Cosmological Physics, University of Chicago, Chicago, IL 60637, USA.

${ }^{g}$ Department of Physics and Astronomy, University of California, Los Angeles, CA 90095, USA.

${ }^{h}$ Physics Department, Vrije Universiteit Brussel, Ixelles, Belgium.

${ }^{i}$ Department of Physics, The Ohio State University, Columbus, OH 43210, USA.

j Jet Propulsion Laboratory, California Institute for Technology, Pasadena, CA 91109, USA.

${ }^{k}$ Max-Planck-Institut für Kernphysik, Heidelberg, Germany.

E-mail: azeolla@psu.edu

When Earth-skimming tau neutrinos interact within the Earth, they generate upgoing tau leptons that can decay in the atmosphere, forming extensive air showers. The Beamforming Elevated Array for COsmic Neutrinos (BEACON) is a novel detector concept that utilizes a radio interferometer atop a mountain to search for the radio emission due to these extensive air showers. The prototype, located at the White Mountain Research Station in California, consists of 4 crossed-dipole antennas operating in the $30-80 \mathrm{MHz}$ range and uses a directional interferometric trigger for reduced thresholds and background rejection. The prototype will first be used to detect down-going cosmic rays to validate the detector model. A Monte-Carlo simulation was developed to predict the acceptance of the prototype to cosmic rays, as well as the expected rate of detection. In this simulation, cosmic ray induced air showers with random properties are generated in an area around the prototype array. It is then determined if a given shower triggers the array using radio emission simulations from ZHAireS and antenna modelling from XFdtd. Here, we present the methodology and results of this simulation.

$37^{\text {th }}$ International Cosmic Ray Conference (ICRC 2021)

July 12th - 23rd, 2021

Online - Berlin, Germany

\footnotetext{
*Presenter
} 


\section{Introduction}

Neutrinos with energies $>10^{17} \mathrm{eV}$ can be produced by astrophysical sources [1] or by the interaction of ultrahigh energy cosmic rays with cosmic background photons [2]. Astrophysical sources produce primarily $v_{e}$ and $v_{\mu}$, however due to flavor mixing the ratio of neutrino fluxes after propagation to Earth is expected to be $v_{e}: v_{\mu}: v_{\tau}=1: 1: 1$ [3]. At these energies, the Earth is opaque to all flavors of neutrino; however, characteristics of the $\tau$ lepton such as its long propagation length and short lifetime provide a unique opportunity for $v_{\tau}$ detection. A $v_{\tau}$ which skims the Earth can interact and produce a $\tau$ lepton which escapes into the atmosphere. The $\tau$ lepton can then decay forming an extensive air shower which emits radio waves due to a combination of geomagnetic emission and the Askaryan effect [4, 5].

The Beamforming Elevated Array for COsmic Neutrinos (BEACON) is a detector concept designed to detect the radio emission created by these upgoing air showers. It consists of phased antenna arrays placed atop mountains. The high elevation sites provide BEACON with large viewing areas which can be monitored almost continuously due to the duty cycles available to ground-based radio antennas. Phased arrays implemented at the trigger level improve the signal-to-noise ratio - thereby lowering trigger thresholds - and allow directional rejection of noise. The BEACON concept is described in greater detail in Reference [6].

A prototype of the BEACON concept is located within the White Mountains near Barcroft Field Station in California [7]. It consists of 4 crossed-dipole antennas operating in the 30-80 MHz range. It uses a phased trigger system modeled after the one used in the ARA experiment [8]. A goal with the prototype is to demonstrate the capability of RF-only triggering on cosmic rays. The flux of cosmic rays is well known so a measurement of the cosmic ray event rate can be used to validate and refine sensitivity estimates and detector models. The cosmic ray search is ongoing with 2 years of data collected thus far [9].

To estimate the acceptance of BEACON to cosmic rays, and from there the detection rate, a Monte-Carlo simulation based on that used by the OVRO-LWA experiment [10] was developed named the Cosmic Ray Simulation for a Beamforming Elevated Array, or Cranberry. In this paper, we will first discuss how Cranberry works, then we will present the estimated acceptance and cosmic ray event rate for the BEACON prototype, and lastly we will discuss future plans for Cranberry and BEACON.

\section{Method}

To calculate the acceptance as a function of cosmic ray energy, Cranberry iterates over a set of randomly-generated showers for each energy bin. The geometry of Cranberry is shown in the left of Fig. 1. Cosmic ray air showers are generated in a circular area around the antenna array. $\cos ^{2} \theta_{z}$ is sampled uniformly from the interval $[0,1]$ such that the cosmic ray zenith angle $\left(\theta_{z}\right)$ lies in the interval $[0, \pi / 2]$, while the azimuth angles $\left(\phi_{C R}\right)$ are uniformly sampled from the interval $[0,2 \pi)$. The position at which the shower intersects the ground is then also randomly chosen.

The beamforming trigger of BEACON allows the instrument to tune the field of view both in azimuthal and polar angles. Many possible mountaintop sites may have a limited field of view because the region overlooking the horizon is occulted by the mountains to the side or behind the 

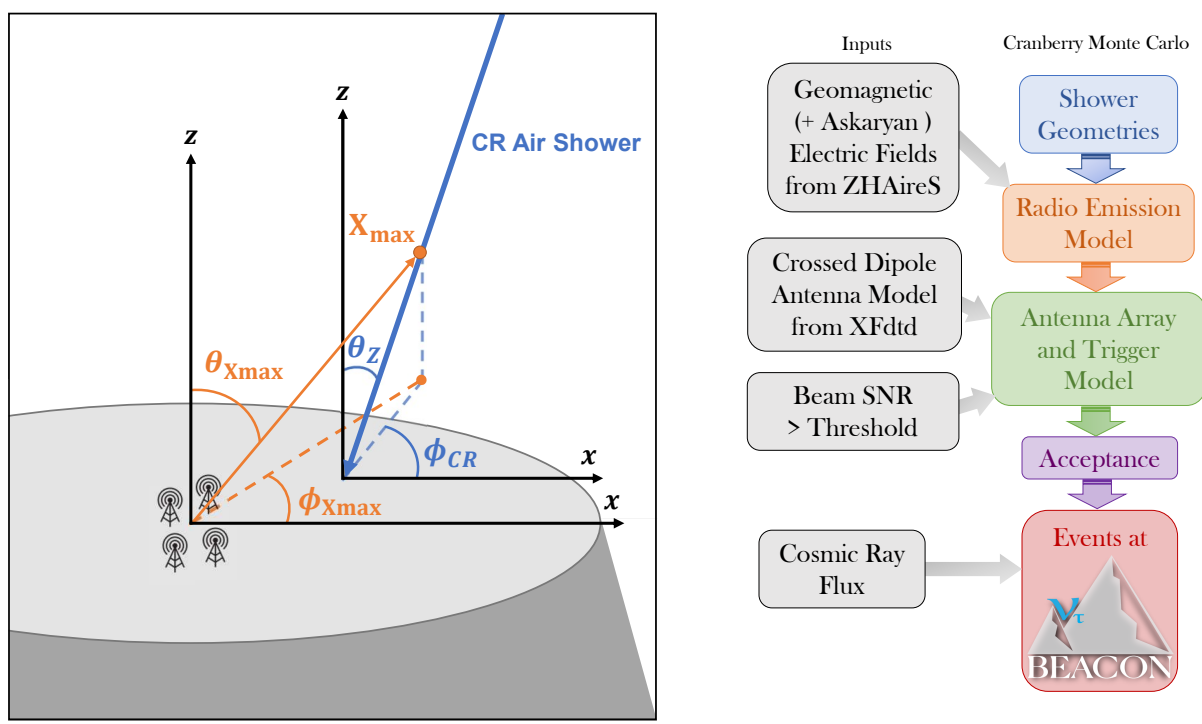

Figure 1: (Left) The geometry of Cranberry. Showers are simulated with randomized zenith $\left(\theta_{z}\right)$ and azimuth $\left(\phi_{C R}\right)$ angles. The positions at which the showers intersect a circular area at the altitude of the BEACON array $(3.875 \mathrm{~km})$ are also randomly chosen. (Right) Flow chart summarizing the procedure of Cranberry that includes the procedural flow of the Monte Carlo on the right and the inputs to the simulation on the left.

array, as is the case at the BEACON prototype site. Because of this, only events thrown within a nominal (but tunable) field of view are allowed to trigger the detector in the Monte Carlo. For this study we used a $120^{\circ}$ field of view $\left(-60^{\circ}\right.$ to $60^{\circ}$ relative to East) to match that expected for the prototype.

The radio emission of each shower at the location of the antenna array is determined using a library of ZHAireS simulations [11]. The library consists of 1,000 simulations with proton primaries at an energy of $10^{17} \mathrm{eV}$. The zenith angles are sampled from a cosine distribution from $30^{\circ}$ to $90^{\circ}$. The minimum zenith angle simulated is $30^{\circ}$, since more vertical showers have an $X_{\max }$ which lies below the BEACON prototype (located at $3.875 \mathrm{~km}$ above sea level) and are unlikely to be detected. Azimuth angles $\left(\phi_{C R}\right)$ are sampled uniformly from the interval $[0,2 \pi]$. For each randomly-generated shower in Cranberry, the simulation with the closest zenith and azimuth angles is chosen from the 1,000 shower ZHAireS library. To test the accuracy of this method, 10,000 random zenith and azimuth angle pairs were created and their best match in the library was found. The standard deviation in the zenith angle error was $0.9^{\circ}$ while the standard deviation in the azimuth angle error was $3.9^{\circ}$.

Once a ZHAireS simulation is chosen from the library, the electric field at the location of the array is determined through interpolation similar to that used in the radio-morphing technique [12]. In each ZHAireS simulation the electric fields are simulated at points in a star-shaped grid in the shower plane. The star-shaped grid has 8 arms of 20 points. The size of each grid is such that the angle between a line extending from the end of an arm to $X_{\max }$ and the shower-axis is $3^{\circ}$ at the elevation of the BEACON prototype. This was chosen because the radio emission $>3^{\circ}$ off-axis is minimal [12]. The electric field at a desired location is determined by first finding the intersection 

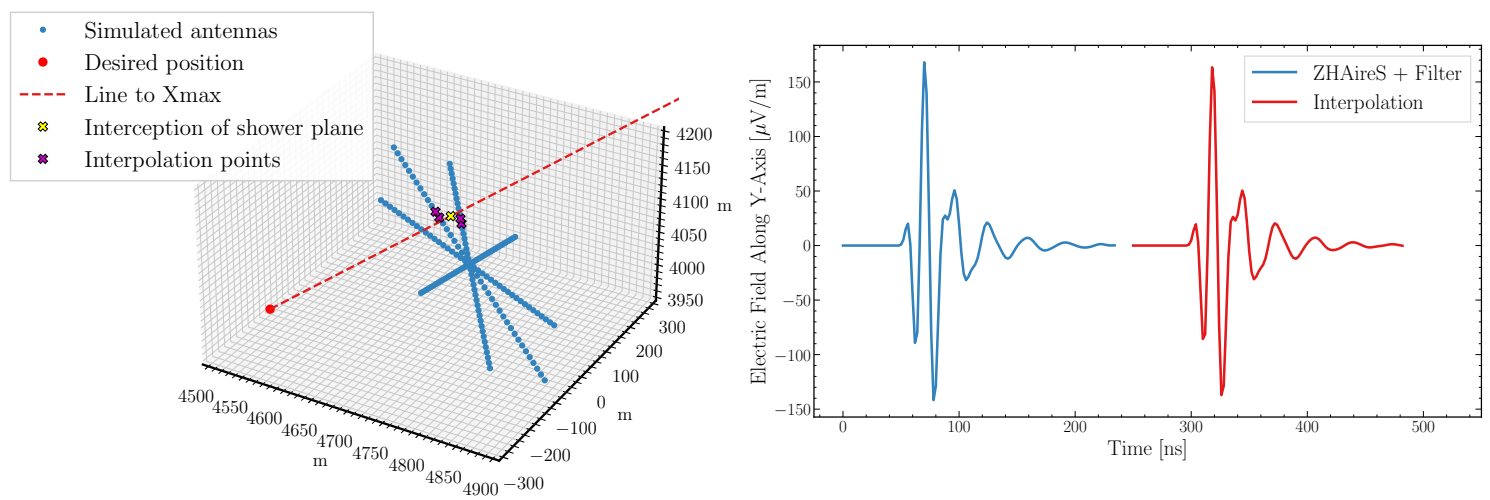

Figure 2: (Left) To determine the electric field at a desired position, the intersection point between a line extending to $X_{\max }$ and the plane of ZHAireS-simulated antennas is found. A bilinear interpolation is then performed on the Fourier-transformed electric fields of the simulated antennas surrounding the intersection point. (Right) A comparison between the filtered ZHAireS-simulated waveform at the desired position versus that obtained through interpolation. The waveforms have been offset in time for comparison.

between a line extending from the location to $X_{\max }$ and the plane of the grid. This is shown on the left in Fig. 2.

A bilinear interpolation is performed using the four grid-points surrounding the intersection point. The electric fields at the grid-points are first filtered to the $30-80 \mathrm{MHz}$ range using a Butterworth filter of order 5, then Fourier transformed. A bilinear interpolation of the real and imaginary components of the frequency-domain signals is then performed. The final signal is then transformed back into the time-domain through an inverse Fourier transform. The signal is then scaled by the distance from $X_{\max }$ to the intersection point over the distance from $X_{\max }$ to the desired location. Finally, the signal is scaled by the ratio of the desired cosmic ray energy to the ZHAireS cosmic ray energy, $10^{17} \mathrm{eV}$. An example of the result of this interpolation procedure is shown on the right in Fig. 2. The blue curve is the filtered ZHAireS waveform at the desired position, while the red curve was found through interpolation. For each shower in the library, 10 test points within the $3^{\circ}$ emission cone were randomly chosen. The percent error between the amplitudes of the interpolated waveform and the ZHAireS-simulated waveform was found for each test point. The standard deviation in percent error was found to be $22.2 \%$ when excluding percent errors greater than $100 \%$ ( $1.5 \%$ of the total). This is comparable to the $27.2 \%$ standard deviation achieved by the radio-morphing technique when excluding outliers [12].

The maximum of the electric field waveform is then found and converted to a voltage. This is done using the antenna impedance and gain as determined by an XFdtd model of the prototype dipole antenna deployed $12-\mathrm{ft}$ above a tilted ground. The gain is directionally dependent and is determined by the vertical and horizontal angle to $X_{\max }$. The voltage is scaled by $N$, the number of antennas, to account for the phased array. The thermal noise observed at the antennas includes contributions from the galactic sky noise (dominant) and the instrument noise dominated by the amplifiers ( $250 \mathrm{~K}$, nominally). A trigger occurs if the ratio of the signal voltage to the noise voltage exceeds a chosen threshold. This is chosen to be $5 \times$ the thermal noise root-mean-squared voltage 

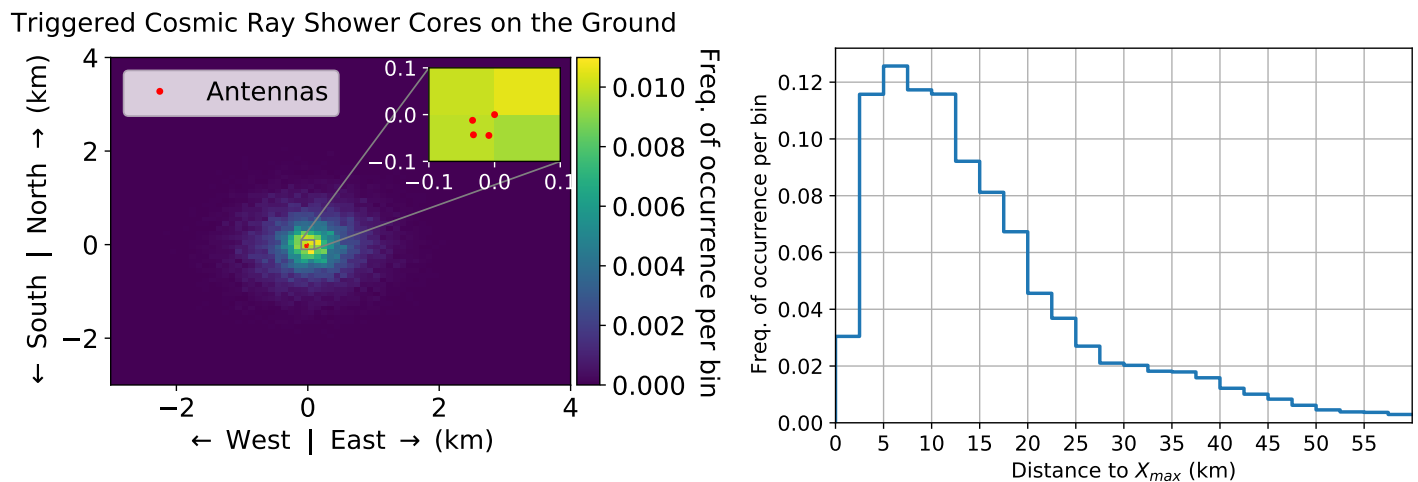

Figure 3: (Left) The locations of the triggered shower cores observed on the ground centered on the BEACON prototype. (Right) The distance from the center of the prototype array to the $X_{\max }$ of triggered events.

$(\sigma)$ nominally. However, given that the instrument adjusts its threshold by continuously monitoring the local radio frequency interference (RFI) to match a global trigger rate, we will study how threshold fluctuations can impact the expected trigger rate.

The acceptance at a given cosmic ray energy is given by

$$
\langle A \Omega(E)\rangle=\iint_{\Omega} d \Omega \iint_{A} d A \hat{n} \cdot \hat{r} P_{o b s}=\pi \times(\text { test area }) \times\left(\frac{\# \text { of showers that trigger }}{\text { total \# of showers }}\right),
$$

where $\hat{n}$ is a vector normal to the test area and $\hat{r}$ is a vector along the shower axis. Once Cranberry has iterated over every randomly-generated shower in each energy bin and found the fraction of showers which induce a trigger we are therefore able to calculate the cosmic ray acceptance as a function of energy. Using the acceptance, known cosmic ray flux, and expected exposure time, one can then calculate the number of expected detected events. The Cranberry procedure is summarized in the flow diagram in the right of Fig. 1.

\section{Results}

We consider in this section the expected cosmic ray results for the BEACON prototype, under several assumptions. The array consists of four dual-polarized electrically-short dipole antennas located at GPS location $\left(37.589310^{\circ} \mathrm{N}, 118.237621^{\circ} \mathrm{W}\right)$ and altitude $3.875 \mathrm{~km}$ within the White Mountain Research Station near Bishop, CA. The magnetic field used in the ZHAireS model of the electric fields is estimated at the center of the array to be (22,294.4 nT North, 4,934.0 nT East, 42,785.2 nT Down) using the IGRF magnetic field model [13]. The two antenna polarizations considered are horizontal, with the antenna tines oriented along the North and South cardinal directions, and vertical, with the antenna tines oriented towards the zenith and the nadir. A trigger is formed if the beamformed peak voltage in the horizontal polarization exceeds a given threshold. We nominally assume a trigger threshold of $5 \sigma$ unless otherwise noted.

The shower core locations that triggered the simulated BEACON prototype are shown in the left of Fig. 3. The prototype array is compact, with the longest baseline being $55 \mathrm{~m}$. Shower cores can still trigger the array up to approximately $1 \mathrm{~km}$ from the center. Because the prototype array is typically at least $2.5 \mathrm{~km}$ away from $X_{\max }$, as shown on the right in Fig. 3, and because the array 

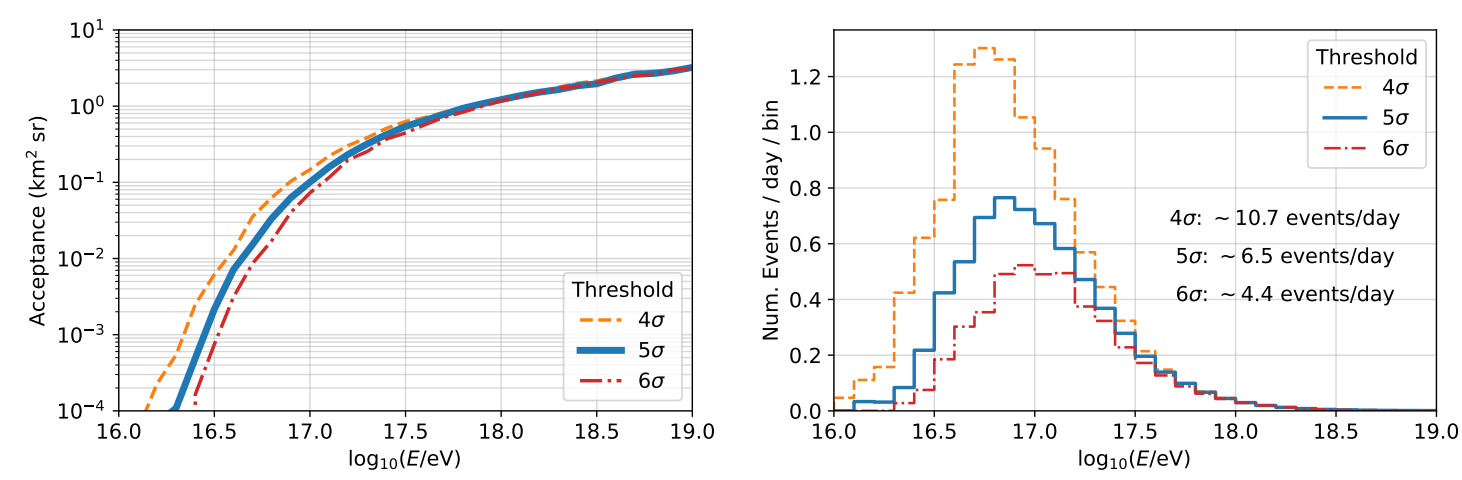

Figure 4: (Left) The acceptance of the BEACON prototype to cosmic ray events and (right) the expected cosmic ray event rate as a function of energy, assuming the cosmic ray flux measured by Auger [14] and assuming different trigger thresholds. Note that while the BEACON array implements a noise-riding trigger, we assume that $5 \sigma$ above the thermal noise voltage is the expected, nominal threshold.

operates in the $30-80 \mathrm{MHz}$ range, the showers are expected to be in the far field and plane-wave like. However, in the on-going cosmic ray search described in these proceedings, events are reconstructed using a spherical wave geometry [9].

The left panel of Fig. 4 shows the expected cosmic ray acceptance for the BEACON prototype, while the right panel shows the expected cosmic ray event rate assuming the cosmic ray flux observed by Auger [14]. We vary the assumed threshold to explore how the local RFI environment affects the expected observed event distributions. At the nominal $5 \sigma$ threshold, the event distribution peaks at $10^{16.8} \mathrm{eV}$. Integrated across all energies we expect to detect $\sim 6.5$ events per day.

The expected angular distributions in both $\theta_{X \max }$ and $\phi_{X \max }$ are shown in Fig. 5. We expect to observe highly inclined cosmic ray air showers due to the high altitude of the observatory and orientation of the antennas. The depth of maximum of $10^{17} \mathrm{eV}$ proton showers arriving at $\theta_{z}$ less than $30^{\circ}$ is below the detector altitude, making the radio signal from those showers weaker. Showers at higher energies reach $X_{\max }$ below the detector at higher zenith angles still. Additionally, the closer $X_{\max }$ is located to the BEACON array, the less likely that the antennas will lie in the $3^{\circ}$ emission cone.

Critically important is the expected rate of cosmic rays near, but above, the horizon, as it sets an upper limit on the expected air shower background for a neutrino search. Out of 260,000 total simulated showers 35,239 induced a trigger, and of those, 14 had an angle $\theta_{X \max }$ within $2^{\circ}$ of the horizon. We can estimate then that the fraction of events near-horizon will be about $4 \times 10^{-4}$. In the event that cosmic rays are detected near the horizon, they can be distinguished from neutrinoinduced upgoing air showers via their polarity, which will further reduce the expected background rate. These results will be folded together with the cosmic ray study to estimate the expected backgrounds for a full-scale BEACON detector in a future study.

The distribution of triggered azimuth angles $\phi_{X \text { max }}$, shown in the right panel of Fig. 5, peaks in the direction of magnetic East, $12.5^{\circ}$ south of geographic East $\left(0^{\circ}\right.$ in the figure). This is consistent with what we expect due to geomagnetic emission. The nulls of the antennas are located at $90^{\circ}$ and $-90^{\circ}$, outside of our $120^{\circ}$ field of view. 

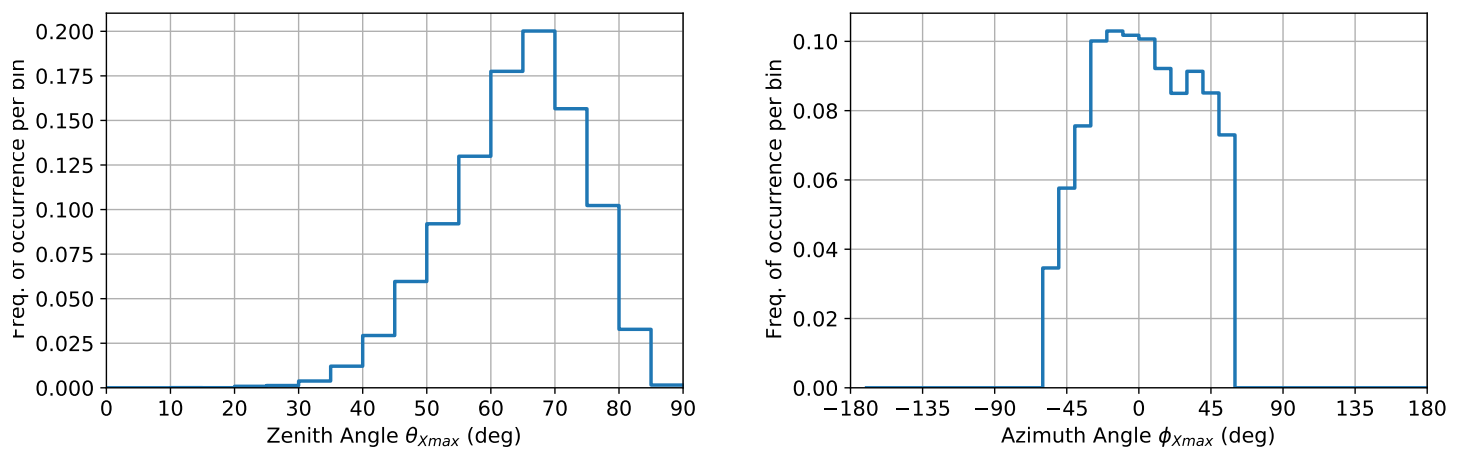

Figure 5: The expected distributions in zenith $\left(\theta_{X \max }\right)$ and azimuth angle $\left(\phi_{X \max }\right)$ of cosmic ray events observed from the BEACON prototype. The left panel illustrates how the high elevation of the site restricts the observed cosmic ray events to be highly inclined. The right panel shows the expected azimuthal distribution assuming a $120^{\circ}$ field of view. The distribution peaks in the direction of magnetic East $\left(-12.5^{\circ}\right)$.

\section{Discussion}

We anticipate several extensions to the current Cranberry model. These improvements are being developed in parallel with the neutrino detector model for BEACON [6] and will therefore inform the development of the future observatory. One important feature of the Cranberry library is that we can extract full time-domain waveforms observed at the antennas of the BEACON prototype. By fully convolving the complex, three-dimensional antenna response, along with the filters and signal chain, we will be able to model the voltages received at the digitizer. These waveforms can then be used for template searches in the cosmic ray analysis.

We plan to upgrade the trigger model to more accurately describe the beamformed trigger based on power sums in the time domain [15]. While it is straightforward to compare the SNR-based trigger and the power sum trigger implemented in the array using simulated electric fields from ZHAireS, we plan to fully incorporate the power sum technique in future iterations of the cosmic ray simulation. Morever, we currently assume a uniform field of view (limited to within $120^{\circ}$ in azimuth). We expect to modify this aspect of the model in the future to incorporate the shape of the phased array beams formed in the trigger of the prototype. Additionally, the full time-domain voltages that trigger within Cranberry can be used to estimate the expected polarizaton angle of the observed cosmic ray events.

Lastly, Cranberry currently assumes a flat ground at an elevation of $3.875 \mathrm{~km}$. In the future, the differing altitudes of the antennas in the BEACON array, as well as the topography of the surrounding mountain range, will be considered to study the effects on the sensitivity of the prototype.

Based on these results, the BEACON prototype is expected to detect multiple highly-inclined cosmic ray events per day, even with fluctuations in the trigger threshold due to local RFI. The results of this simulation will be used to guide the ongoing analysis of data collected by the prototype array, which will be the first search of this type from this geometry.

This work is supported by NSF Awards 2033500, 1752922, 1607555, \& DGE-1746045 as well as the Sloan Foundation, the RSCA, and NASA (support through JPL and Caltech as well as Award 80NSSC18K0231). This work has received financial support from Xunta de Galicia (Centro singular 
de investigación de Galicia accreditation 2019-2022), by European Union ERDF, by the "María de Maeztu" Units of Excellence program MDM-2016-0692, the Spanish Research State Agency and from Ministerio de Ciencia e Innovación PID2019-105544GB-I00 and RED2018-102661-T (RENATA). We thank the NSF-funded White Mountain Research Station for their support.

\section{References}

[1] E. Waxman and J. Bahcall, High energy neutrinos from astrophysical sources: An upper bound, Physical Review D 59 (1998) .

[2] V. Beresinsky and G. Zatsepin, Cosmic rays at ultra high energies (neutrino?), Physics Letters B 28 (1969) 423.

[3] J.F. Beacom, N.F. Bell, D. Hooper, S. Pakvasa and T.J. Weiler, Measuring flavor ratios of high-energy astrophysical neutrinos, Phys. Rev. D 68 (2003) 093005.

[4] E. Zas, Neutrino detection with inclined air showers, New Journal of Physics 7 (2005) 130.

[5] F.G. Schröder, Radio detection of cosmic-ray air showers and high-energy neutrinos, Progress in Particle and Nuclear Physics 93 (2017) 1-68.

[6] S. Wissel et al., Prospects for high-elevation radio detection of $>100 \mathrm{PeV}$ tau neutrinos, JCAP 11 (2020) 065 [2004 . 12718].

[7] K. Hughes et al., Towards interferometric triggering on air showers induced by tau neutrino interactions, PoS ICRC2019 (2020) 917.

[8] ARA collaboration, A ground-based interferometric phased array trigger for ultra-high energy neutrinos, PoS ICRC2017 (2018) 1013.

[9] D. Southall et al., Searching for rf-only triggered cosmic ray events with the high-elevation beacon prototype, PoS ICRC2021.

[10] R. Monroe et al., Self-triggered radio detection and identification of cosmic air showers with the OVRO-LWA, NIM-A 953 (2020) 163086.

[11] J. Alvarez-Muñiz, W.R. Carvalho and E. Zas, Monte Carlo simulations of radio pulses in atmospheric showers using ZHAireS, Astroparticle Physics 35 (2012) 325-341.

[12] A. Zilles et al., Radio morphing: towards a fast computation of the radio signal from air showers, Astroparticle Physics 114 (2020) 10-21.

[13] P. Alken et al., International geomagnetic reference field: the thirteenth generation, Earth, Planets and Space 73 (2020) .

[14] Pierre Auger collaboration, The cosmic ray energy spectrum measured using the Pierre Auger Observatory, PoS ICRC2017 (2018) 486.

[15] P. Allison et al., Design and performance of an interferometric trigger array for radio detection of high-energy neutrinos, Nucl. Instrum. Meth. A 930 (2019) 112 [1809. 04573]. 